\title{
Study on the generation of Efficient Terahertz from cavity phase matching optical parametric oscillator with a GaAs Crystal sheet by $\mathrm{CO}_{2}$ laser
}

\author{
Zhi-Ming Rao ${ }^{1, a^{*}}$, Hui-Hui Xu ${ }^{1, b}$ and Fang-Sen $\mathrm{Xie}^{1, \mathrm{c}}$ \\ ${ }^{1}$ School of Physics, Communication and Electronics, Jiangxi Normal University, nanchang, Jiangxi, \\ 330022, CHINA

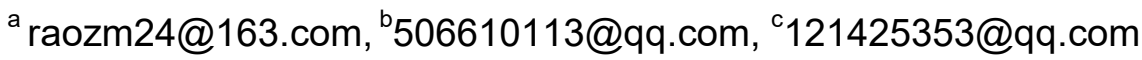 \\ * corresponding author : raozm24@163.com
}

Keywords: terahertz, cavity phase matching, optical parametric oscillator, GaAs crystal sheet.

Abstract. We study on the generation of efficient terahertz (THz) wave. This scheme applying cavity phase matching (CPM) optical parametric oscillator (OPO) with a $\mathrm{GaAs}$ crystal sheet by $\mathrm{CO}_{2}$ laser is proposed. The $\mathrm{THz}$ wave is emitted the nonlinear crystal. We calculated the optimized cavity length in a GaAs crystal sheet at the range of 100-1000 $\mu \mathrm{m}$. The result is shown that CPM-OPO scheme is efficient to obtain efficient $\mathrm{THz}$ wave.

\section{Introduction}

Terahertz (THz) sources can be used in many applications in environment detections, security inspections, biomedical diagnostics, and so on [1-3]. Among the variety of nonlinear crystals, GaAs meet the requirements for high transparency in both $10 \mu \mathrm{m}$ band and relatively high transparency in the $\mathrm{THz}$ region. A lot of work has been carried out enhancing $\mathrm{THz}$ power [[4-7]. We demonstrate a tunable THz source based on the collinear DFG in GaSe crystal with a dual-wavelength $\mathrm{CO}_{2}$ laser [7]. A peak power of $182 \mathrm{~mW}$ was detected, which peak power corresponds to be external power conversion efficiency only about $1 \times 10^{-5} \%$. Optical parametric oscillation(OPO) is one of the effective ways to produce high-power, narrow-band coherent $\mathrm{THz}$ sources in nonlinear crystals[4]. Optical parametric oscillation(OPO) was designed to satisfy the wave vector phase matching condition in nonlinear crystal. The scheme of cavity phase matching using has been proposed [8, 9] and demonstrated [10] to solve nonlinear optical crystals high absorption coefficients. In this scheme, the $\mathrm{THz}$ wave is generated in cavity phase matching. CPM-OPO makes it possible to avoid $\mathrm{THz}$ wave strongly absorbing crystals decay.

\section{Theory of scheme and discussion}

GaAs crystal has a large nonlinear coefficient, a widely transparency range, and a high damage threshold. According to the Manley-Rowe relation, the maximum conversion efficiency can be improved by one order of magnitude when using mid-infrared laser with longer wavelength running at $10 \mu \mathrm{m}$. Taking into account the maturity of mid-infrared laser technology and the development of technology of periodically-inverted crystals, it will be an effective way to generate $\mathrm{THz}$ wave based on CPM-OPO. However, the theory generation of the efficient $\mathrm{THz}$ wave in a crystal sheet cavity phase matching pumped by wavelength running at $10 \mu \mathrm{m}$ laser has not been detail reported.

In this letter, explore the terahertz (THz) wave generation based on cavity phase matching (CPM) Optical parametric oscillation in a GaAs crystal sheet. It is found that the Zinc blende semiconductor GaAs is an efficient $\mathrm{THz}$ frequency generator owing to its large second-order nonlinear coefficient $\left(\mathrm{d}_{36}(10.6 \mu \mathrm{m})=83 \mathrm{pm} / \mathrm{V}\right)[11]$. Our scheme is cavity phase matching (CPM). The optimized cavity length in a GaAs crystal sheet were calculated in this paper.

The geometry principle of phase matching is as shown. Here we assume that the wave propagation of the pump wave (at frequency $\omega_{P}$, wavelength $\lambda_{P}$ ) is perpendicular to the domain wall of the nonlinear crystal. The THz wave (at frequency $\omega_{T}$, wavelength $\lambda_{T}$ ) is emitted the nonlinear 
crystal. The damping of the $\mathrm{THz}$ wave is minimized due to the short pass length in the nonlinear crystal. The optimized cavity length in a GaAs crystal sheet $\Lambda$ can be calculated by using the vector phase matching condition and energy conservation:

$$
\begin{aligned}
& k_{P}=k_{S}+k_{T}+\frac{\pi}{\Lambda}, \\
& \frac{1}{\lambda_{P}}=\frac{1}{\lambda_{T}}+\frac{1}{\lambda_{S}},
\end{aligned}
$$

where, $k_{i}=2 \pi n_{i} / \lambda_{i}$ and $n_{i}(i=P, S, T)$ denote pump wave, singal wave and $\mathrm{THz}$ wave vector and refractive indices, respectively. The grating period $\Lambda$ can be deduced from equations (1) and (2)

$$
\Lambda=\frac{\lambda_{P} \lambda_{T}}{2\left(n_{P} \lambda_{T}-n_{T} \lambda_{P}-\lambda_{T} n_{S}+\lambda_{P} n_{S}\right)},
$$

This scheme can produce THz wave. For $\lambda_{P}=9.552 \mu \mathrm{m}$, we calculated optimized cavity length in a GaAs crystal sheet $\Lambda$ by using the Sellmeier equation of GaAs from Ref. [12] and Eq. (3). The Sellmeier equation of GaAs is given by

$$
n^{2}(\lambda)=b+\frac{g_{1}}{b_{1}^{-2}-\lambda^{-2}}+\frac{g_{2}}{b_{2}^{-2}-\lambda^{-2}}+\frac{g_{3}}{b_{3}^{-2}-\lambda^{-2}}
$$

where, $\lambda$ is the pump or Thz laser wavelength, $b=5.372514, b_{1}(\mu \mathrm{m})=0.4431307+0.000050564 \Delta \mathrm{T}$, $\mathrm{b}_{2}(\mu \mathrm{m})=0.8746453+0.0001913 \Delta \mathrm{T}-4.882 \times 10^{-7} \Delta \mathrm{T}^{2}, \quad \mathrm{~b}_{3}(\mu \mathrm{m})=36.9166-0.011622 \Delta \mathrm{T}, \quad \Delta \mathrm{T}$ is the deviation from the reference room temperature $22^{\circ} \mathrm{C}, \quad \mathrm{g}_{1}=27.83972$, $\mathrm{g}_{2}=0.031764+4.350 \times 10^{-5} \Delta \mathrm{T}+4.664 \times 10^{-7} \Delta \mathrm{T}^{2}$, and $\mathrm{g}_{3}=0.00143436$.

The relation of the optimized cavity length in a GaAs crystal sheet $\Lambda$ at room temperature and the THz wavelength are shown in Fig. 1 from Eqs.(3) and (4). It can be seen that $\Lambda$ is increased from 0.16 to $1.96 \mathrm{~mm}$ when $\mathrm{THz}$ wavelength is increased from 100 to $1000 \mu \mathrm{m}$ in the CPM scheme. Needing the optimized cavity length in a GaAs crystal sheet is a $0.58 \mathrm{~mm}$ in the CPM scheme to generate a frequency of 1

$\mathrm{THz}$ $(300 \mu \mathrm{m})$ wave.

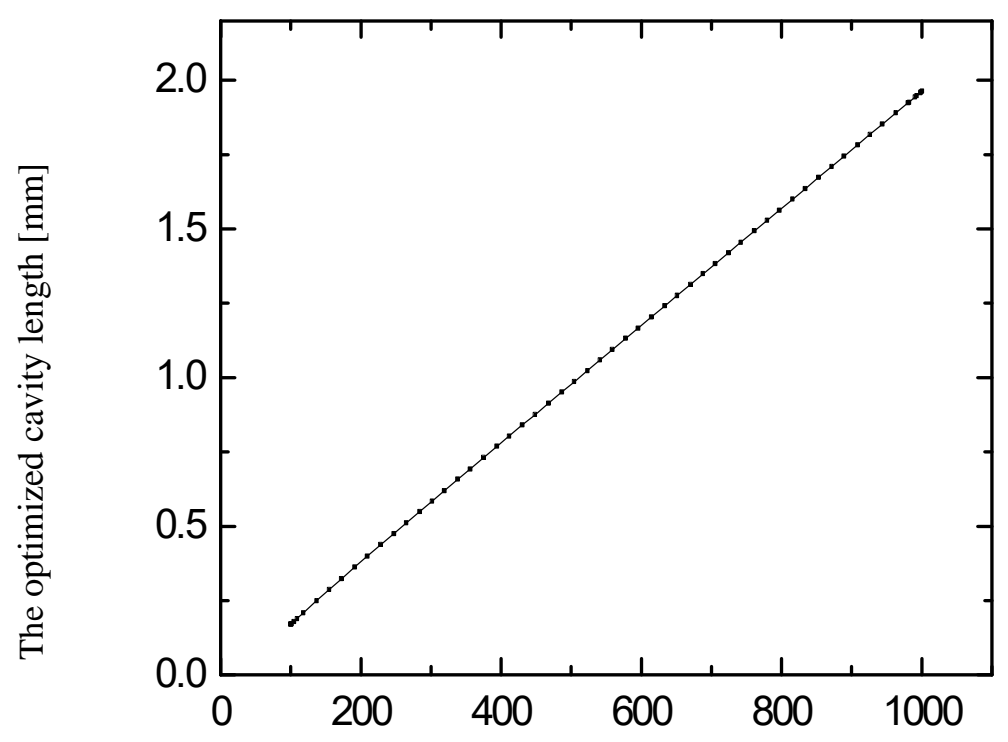

THz wavelength[ $[\mu \mathrm{m}]$

Fig.1. The optimized cavity length versus the generated $\mathrm{THz}$ wavelength with a GaAs crystal sheet 
To evaluate the performances for using crystals as THz-wave converters, all the interactions can be phase matched in OPO, and $\mathrm{THz}$ power conversion efficiency $\eta$ in the plane-wave fixed-field can be given by [13]

$$
\eta=\frac{8 \pi^{2} d_{e f f}^{2} L^{2} P_{P}}{\varepsilon_{0} c n_{1} n_{2} n_{T} \lambda_{T}^{2} A} e^{-\alpha_{T} L},
$$

where, $L$ is the crystal length $0.058 \mathrm{~cm}$, the pump intensity $P_{\mathrm{P}}=10 \mathrm{MW}$, dielectric constant of vacuum $\varepsilon_{0}=8.854187817 \times 10^{-12} \mathrm{~F} / \mathrm{m}$, light speed of vacuum $\mathrm{c}=3 \times 10^{8} \mathrm{~m} / \mathrm{s}$, and the pump area $A=0.1 \mathrm{~mm}^{2}$. Zinc blende semiconductor GaAs THz absorption coefficient $\alpha_{\mathrm{T}}$ is $0.5-4.5 \mathrm{~cm}^{-1}$ at $1-3 \mathrm{THz}$ [7]. For OPO process, when $\mathrm{THz}$-wave $\lambda_{T}=300 \mu \mathrm{m}(1 \mathrm{THz})$ from Eq. (5), which corresponds to the power conversion efficiency is of $\sim 0.000001 \%$.

In this CPM- OPO scheme, to evaluate the performances for using crystals as $\mathrm{THz}$-wave converters, assume that the pump wave achieves 10 times in a GaAs crystal sheet. THz-wave power conversion efficiency $\eta$ neglecting pump depletion in the plane-wave fixed-field can be given by

$$
\eta=\frac{3200 d_{\text {eff }}^{2} L^{2} P_{P}}{\varepsilon_{0} c n_{1} n_{2} n_{T} \lambda_{T}^{2} A},
$$

where, $b=0.3 \mathrm{~mm}$ is the crystal thickness, and the overlap factor $F=1$. For the CQPM scheme, at THz-wave $\lambda_{T}=100 \mu \mathrm{m}(3 \mathrm{THz})$ from Eq.(6), which corresponds to the power conversion efficiency of $\sim 0.00005 \%$. However, the power conversion efficiency of THz-wave at $3 \mathrm{THz}$ is only $0.000001 \%$ in DFG. In CPM- OPO, the power conversion efficiency of THz-wave is far more than that of in DFG, which is due to the CPM- OPO pump wave achieves 10 times in a GaAs crystal sheet.

\section{Summary}

In conclusion, we have investigated $\mathrm{THz}$ generation from the optimized cavity length in a GaAs crystal sheet with wavelength running at $10 \mu \mathrm{m}$, and scheme of phase matching is CPM pattern. Using CPM- OPO the pump wave achieves 10 times in a GaAs crystal sheet, and the power conversion efficiency is $0.00005 \%$ at a frequency of $1 \mathrm{THz}$, since the pump beam is achieve 10 times in a GaAs crystal sheet. We calculated the optimized cavity length in a GaAs crystal sheet at $\mathrm{THz}$ wavelength from 100 to $1000 \mu \mathrm{m}(0.3-3 \mathrm{THz})$. It is shown that the optimized cavity length in a GaAs crystal sheet is efficient to obtain high power conversion efficiency of $\mathrm{THz}$ wave generation with mid-infrared laser by using CPM- OPO.

\section{Acknowledgements}

This work was financially supported by the Natural Science Fundation of Jiangxi Province (20161BAB202052), by the Science Fundation of Jiangxi Province Office of Eduaction (150337), and by the Fundation of Jiangxi Normal University (7170).

\section{References}

[1] Y.M. Bahk, H.R. Park, K.J. Ahn, H.S. Kim, Y.H. Ahn, D.S. Kim, J. Bravo-Abad, L.

Martin-Moreno, and F.J. Garcia-Vidal, Phys. Rev. Lett. Vol.106 (2011), 013902.

[2] M. B. Johnson, D.M. Whittaker, et al, Linfield: Phys. Rev. B. Vol. 65(2002), 165301.

[3] X. Xie, J. Dai, Zhang. X.C, Phys. Rev. Lett. Vol.96 (2006), 075505.

[4] Z.M. Rao, X.B. Wang and Y. Z. Lu, Optics Communications, Vol.284(2011),5472-5474.

[5] Z.M. Rao, X.B. Wang, Y. Z. Lu, et al, Chinese Physics Letter, Vol.28(2011), 074215.

[6] Z.M. Rao, W. Tao, Y. He, et al, SPIE, Vol.8330(2011), 83300Q. 
[7] Y. Geng, X. Tan, X. Li, J. Yao, Compact and widely tunable terahertz source based on a dual-wavelength intracavity optical parametric oscillation, Appl. Phys. B. Vol. 99 (2010),181-185.

[8] Armstrong J A, Blombergen N, Physics Review, Vol.127(1962), 1918-1939.

[9] Haidar R, Forget N, and Rosencher E,Quantum Electron, Vol.39 (2003) ,569-575.

[10] Xie Z D, Lv X J,Liu Y H, et al,Physical Review Letters, 106(2011):083901.

[11] D. N. Nikogosyan: Nonlinear Optical Crystals: A Complete Survey (New York: Springer) 2005, p208

[12] T. Skauli, P.S. Kuo, K.L. Vodopyanov, T.J. Pinguet, O. Levi, L.A. Eyres, J.S. Harris, B. Gerard, L. Becouarn, and E. Lallier: J. Appl. Phys.Vol.94(2003), p.6447.

[13] V. G. Dmitriev: Handbook of Nonlinear Optical Crystals (Springer publications, Berlin 1999) p50 